\title{
Comptes à rendre et contes rendus
}

La parole donnée au romancier antillais

\section{Anna Lesne}

\section{(2) OpenEdition}

Journals

Édition électronique

URL : https://journals.openedition.org/clo/1699

DOI : $10.4000 /$ clo. 1699

ISSN : 2266-1816

Éditeur

INALCO

Édition imprimée

Date de publication : 2 janvier 2012

ISBN : 978-2-85831-212-2

ISSN : 0396-891X

Référence électronique

Anna Lesne, "Comptes à rendre et contes rendus », Cahiers de littérature orale [En ligne], 72 | 2012, mis en ligne le 29 avril 2015, consulté le 01 juillet 2021. URL : http://journals.openedition.org/clo/1699 ; DOI : https://doi.org/10.4000/clo.1699

Ce document a été généré automatiquement le 1 juillet 2021.

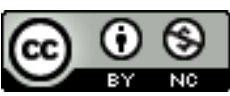

Cahiers de littérature orale est mis à disposition selon les termes de la Licence Creative Commons Attribution - Pas d'Utilisation Commerciale 4.0 International. 


\title{
Comptes à rendre et contes rendus
}

\author{
La parole donnée au romancier antillais
}

\author{
Anna Lesne
}

1 Avec cette apostrophe, la narratrice de la Grande drive des esprits, le premier roman de la Guadeloupéenne Gisèle Pineau, convoque autour d'elle un cercle d'auditeurs, accrochés par une ruse : le dévoilement de cette histoire à mi-chemin entre l'indiscrétion et le conte est une faveur, qu'ils ne pourront sans doute s'empêcher de faire à leur tour. Elle mentionne l'existence de plusieurs versions, questionnant le statut de vérité de son histoire et se déchargeant de toute autorité en la matière, comme le font bien des conteurs: "Peut-être croyez-vous qu'il s'agit là d'affabulations et que cette scène hâtivement brossée ne reflète point la vérité vraie. C'est ainsi qu'on me l'a narrée. Soyez certains que pas une virgule, pas une parole, pas même une marinade n'a été retranchée ou apportée " (ibid., 110). Ces quelques lignes sont un exemple révélateur de l'importance du modèle du conte dans le roman antillais contemporain et d'un paradoxe : la visibilité de l'acte de narrer et du narrateur, et son effacement comme auteur.

\section{Le romancier et la voix collective : questions de légitimité}

2 En 1979, le Ti-Jean L'Horizon de la Guadeloupéenne Simone Schwarz-Bart s'inspire directement et explicitement de la tradition orale, tant pour le contenu que pour le ton et les apostrophes au lecteur; Xavier Orville, le plus latino-américain des romanciers martiniquais, régale ses lecteurs de merveilleux antillais, Ernest Pépin élabore son premier roman, L'Homme-au-bâton, à partir d'un véritable conte urbain né dans la Guadeloupe des années $1950^{1}$ et Raphaël Confiant ravit la critique parisienne qui veut voir dans les romans antillais autant de contes "drolatiques", "savoureux» ou «sensuels»; se donnant pour un «passeur d'imaginaire qui imprime sa marque personnelle sur la trame ininterrompue de l'oraliture»(Confiant, 1995b, 12-13), il transcrit des contes traditionnels, consacre des ouvrages à des conteurs antillais et écrit des contes, comme Patrick Chamoiseau, l'autre nom célèbre du mouvement de la 
Créolitée . Après Manman Dlo contre la fée Carabosse, présenté comme du théâtre conté, celui-ci introduit son premier roman, Chronique des sept misères, par un retentissant "Messieurs et dames de la compagnie», qui l'apparente de toute évidence au conte. Vingt-cinq ans plus tard, le Papillon et la lumière est une variante de « La Conférence des papillons ", conte soufi repris par Henri Gougaud... Paradoxalement, ces champions du roman apparaissent comme les meilleurs défenseurs d'un renouveau du conte aux Antilles.

3 Si la présence du merveilleux antillais et le ton de ces romans, teintés d'oralité, s'accordent à l'horizon d'attente du public français, ces hommages explicites au genre du conte et à la pratique des conteurs correspondent aussi aux préoccupations principales du milieu littéraire guadeloupéen et surtout martiniquais des années 1990, un milieu alors dominé par le mouvement de la créolité. Comme en témoigne l'ouvrage collectif Écrire la «parole de nuit » paru en 1994, la question d'une littérature antillaise plus authentique et de ses liens avec «l'oraliture ${ }^{3}$ est en effet alors centrale, dans une prise de distance réaffirmée avec la tradition littéraire française - question ancienne déjà soulevée par Jean Price-Mars dans les années 1920. L'écriture littéraire apparait comme une pratique culturelle empruntée à l'extérieur et tournée vers l'extérieur, que les écrivains antillais doivent, pour gagner en légitimité, se réapproprier, en tentant de combler le fossé qui s'est établi entre la littérature orale en créole et la littérature écrite en français. Les écrivains antillais se trouvent sommés de rendre des comptes, de se justifier sur leur pratique, et chargés d' "écrire une parole ", en français qui plus est, double traduction ou double trahison. C'est dans cette « anxiété de traîtrise » face à des missions contradictoires et dans le caractère problématique du statut de l'écrivain aux Antilles que Lydie Moudileno (1997) et Dominique Chancé (2000) voient la cause de la mise en scène fréquente, à partir de la fin des années 1980, de personnages d'écrivains dans la fiction antillaise, personnages confrontés à de sévères difficultés, voire à des destins tragiques. Un discours élaboré sur leur pratique et leurs échecs envahit la fiction (notamment chez Chamoiseau) et se développe en marge, dans des essais, entretiens et articles de presse, tandis que le conteur créole, mythifié, se voit accorder un fort indice de légitimité.

Édouard Glissant et les écrivains de la Créolité font du conteur un agent essentiel de la fondation de la société créole. La plantation est un univers caractérisé par la mise en contact de populations étrangères (Européens, esclaves de divers pays d'Afrique, puis engagés d'Inde et de Chine) et donc par la confrontation de systèmes de valeurs et de références hétérogènes; c'est aussi un milieu marqué par des conduites individualistes encouragées par le maître, qui s'efforce d'empêcher ou de distendre les liens de solidarité ; au sein de cet univers, le rôle du conteur est interprété comme central dans la création de lien social, dans l'émergence d'une conscience collective. Selon Glissant, il est celui «qui nous a donné le Nous » $(1981,264)$. «Le Conteur, écrit également Chamoiseau, né d'un désordre d'hommes et tout projeté dans les liens à créer, est inventeur de peuple» $(1997 \mathrm{a}, 187)$. Dans le cercle assemblé le soir à la veillée, «le Conteur fait parler-ensemble ces corps restaurés par les gestes, répondre-ensemble, marcher d'un pas commun, éprouver les mêmes joies, des peurs unanimes, des échappées conjointes. [...] le Conteur parle avec!» (ibid., 186). Les individus rassemblés deviennent des répondeurs : «Ee krii! Ee Kraa!». «En répondant Ee kraa, ce groupe hétéroclite, d'origines culturelles diverses, dit en réalité Nous. C'était crier une cohésion en pleine diversité quasi indéchiffrable» (Chamoiseau et 
Confiant, 1991, 80-81). Dans cette parole donnée, dans cette obligation d'écouter et de répondre, les individus font groupe.

5 Soulignons que ce n'est pas le public et encore moins le maitre de la plantation qui donne la parole au conteur, comme un adulte donnerait la parole à un enfant : « qu'estce que j'ai à dire et qui m'a baillé [donné] la parole? » dit le conteur Solibo dans Solibo Magnifique, de Chamoiseau :

[...] personne personne ne m'a baillé la parole et je n'ai rien à dire je dis la parole c'est tout sans commandeur géreur atron chef et capitaine fout' la parole sans devant ni derrière merde au nègre à qui l'on a baillé la parole par-ici [...] si on lui baille la parole Solibo n'a plus de paroles.

(1988a, 219).

6 Il a besoin en revanche qu'elle soit reçue ; «Ô amis, merci de la faveur, la parole est cueillie » dit Solibo (206) qui, privé d'oreilles, n'est plus qu'un vieux fou soliloquant. Une prise de parole entraîne l'autre, les conteurs se succèdent au centre du cercle, parfois pour s'affronter en de véritables joutes oratoires: les conteurs sont les "Majolè ", les majors de l'air c'est-à-dire les combattants de l'air. Il faut donner un conte et le donner mieux que l'orateur précédent. L'improvisation, la performance personnelle et l'interaction avec l'auditoire caractérisent ce temps collectif, fondateur sur le plan social. Cette pratique est également perçue comme fondatrice sur le plan culturel, par la reprise et la recomposition des "paroles" des uns et des autres d'origines diverses sur la plantation, leur savoir narratif, «l'emmêlée des histoires venues de tous les Territoires» (Chamoiseau, 1997a, 193). Dans leur essai sur l'histoire littéraire antillaise, Lettres créoles (1991), Chamoiseau et Confiant font ainsi du conteur le «Papa » de la «tracée » majeure de la littérature créole, plaçant l'oraliture et la pratique du contage au cœur des recherches sur une poétique antillaise contemporaine.

7 En référence à la situation d'énonciation du conteur, Chamoiseau introduit dans certains textes des "répondeurs »: l'un de ses récits d'enfance romancés, Chemin d'école, est entrecoupé d'appels et de réponses - «Je demande les Répondeurs, à présent... », «Répondeurs: On t'entend! On t'entend !...» $(1994 c, 18,21)$. Ailleurs, il introduit un dialogue avec des commentateurs, par exemple ce « vieux guerrier » qui relance sa réflexion dans son essai Écrire en pays dominé. Dans d'autres textes encore, il mêle sa voix à celle de ses pairs, intercalant un "entre-dire " de Glissant entre les chapitres de l'Esclave vieil homme et le molosse, co-signant des essais avec Confiant et deux autres avec Glissant. Il est ainsi frappant de constater qu'aucun de ses textes, même ses récits d'enfance et de formation, ne semble faire entendre sa voix seule. Non seulement le romancier souligne dans ses romans une forte dimension dialogique mise en évidence entre autres par des jeux intertextuels, mais, par la mise en scène explicite d'interlocuteurs imaginaires ou réels, il nie être " confiné dans son isolement », pour reprendre les mots de Walter Benjamin (1936). Glissant, avant lui, cherchait aussi la synthèse "de la solitude d'écriture et de la participation au chanter commun" $(1981,440)$, espérant pouvoir annoncer une «nouvelle nuit des cases dans laquelle nous rassemblons, par chuchotements d'abord, raclés au fond des gorges, nos voix " (1981, 754-755).

8 La volonté de reproduire la posture et la situation d'énonciation du conteur est en relation avec la mise en cause de l'autorité (auctorialité) et de la position de maîtrise de l'auteur, qui associe son texte au discours du maître. Le conteur n'est pas « un créateur en suspension, mais bien le délégué d'un imaginaire collectif auquel son art s'ajoute » (Chamoiseau et Confiant, 1991, 80). "L'auteur ", écrit Glissant dans un chapitre de son 
Discours antillais sur ce que pourrait être le "roman des Amériques", "doit être démythifié, oui, parce qu'il doit être intégré à une décision commune. Le Nous devient le lieu du système génératif, et le vrai sujet » $(1981,442)$. Cependant ce «nous » est disjoint, fait d'une multiplicité de voix singulières qui ne se rejoignent qu'occasionnellement. Dans les romans de Glissant, le récit est éclaté et polyphonique, pris en charge par ses personnages; il ne s'agit pas tant de raconter une histoire, commente Dominique Chancé, que de se demander qui raconte, de quel point de vue, avec quelle autorité, quel savoir; l'acte de narrer devient l'enjeu même du récit (Chancé, 2000, 15). Chez Chamoiseau, à l'unité énonciative du premier roman, Chronique des sept misères (conté par un "nous»), succède un chahut de voix singulières dans Solibo Magnifique. S'en détache celle du « Marqueur de paroles », expression par laquelle Glissant a désigné l'auteur de Chronique des sept misères dans une préface à sa réédition en 1988, et personnage-clé des romans suivants de Chamoiseau - produit d'une réflexion théorique sur le passage de l'oral à l'écrit, du créole au français, et sur la position possible ou acceptable de l'écrivain aux Antilles françaises.

Chamoiseau élabore à partir de Solibo Magnifique la figure d'un écrivain qui "écrit» moins, dans le sens d'une écriture créative, qu'il ne transcrit, traduit, dialogue; il recueille des paroles et les met par écrit, les «marque» dans un premier sens du terme; il réagit aussi en présence des voix du groupe comme peut le faire le «marqueur» du gwoka - ce genre musical guadeloupéen qui est avec le contage une autre pratique mythifiée des veillées sur la plantation: le tambour soliste suit les pas $\mathrm{du}$ danseur, les traduit en phrases rythmiques et relance le «dialogue ». Dans Solibo Magnifique, Chamoiseau met en scène la mort du conteur (le dénommé Solibo qui, très symboliquement, s'étrangle en pleine performance), la récupération de sa parole par le Marqueur de paroles et le procès intenté à l'écrivain. Interrogé par la police qui le suspecte d'avoir tué le conteur, ce personnage refuse d'être qualifié d'écrivain: "Ça change tout, inspectère, l'écrivain est d'un autre monde, il rumine, élabore ou prospecte, le Marqueur refuse une agonie: celle de l'oraliture, il recueille et transmet» (1988a, 159).

\section{Recueillir et transmettre : le conteur, l'ethnographe et le Marqueur de paroles}

10 Le conteur était "gardien des mémoires" (Chamoiseau et Confiant, 1991, 81). Dans L'Esclave vieil homme et le molosse, il est dit du Marqueur de paroles qu'il est « un gardien du passé », "bailleur de nostalgies des âges et des époques, des certitudes et des identités », par lequel «antan [est] restitué au pays» (Chamoiseau, 1997b, 141-142). Dans la même perspective, la quatrième de couverture de Chronique des sept misères présente le roman comme "à la fois une fiction d'une surprenante verve inventive et un document incomparable sur une civilisation en train de disparaître à tout jamais ». En annexe, l'auteur insère une "note de l'ethnographe " déplorant que "seul l'ethnographe pleure les ethnocides insignifiants » (Chamoiseau, 1986, 217) ; est-il luimême cet "ethnographe "? Le personnage du Marqueur de paroles dans Solibo, très explicitement identifié dans un jeu de brouillage réel/fiction avec Chamoiseau, précise être l'auteur de Chronique des sept misères, texte qualifié d'« ethnographie des djobeurs du marché » : le narrateur se donne à voir équipé de cahiers et d'un magnétophone qui ne fonctionne jamais, caractérisé par une "appétence malsaine pour les paroles [...] 
même les plus inutiles » et s'imaginant "en observation directe participante, comme le douteux Malinowski, Morgan, Radcliffe-Brown, ou bien Favret-Saada chez ses sorciers normands » (1988a, 42-43). Le Marqueur de paroles, double romanesque de l'écrivain, s'est ainsi «prétendu ethnographe »; d'ailleurs l'épigraphe de Solibo Magnifique est un très court échange entre "l'ethnographe » et "le conteur", qu'il appelle "Papa ». Notons que ces différentes identifications proposées par l'auteur à la fois dans la fiction et hors texte ont été redoutablement efficaces dans la réception des romans de Chamoiseau : la critique s'est plu assez systématiquement à faire référence à l'auteur comme à un Marqueur de paroles (Chancé, 2000, 99 sq), jusqu'à l'accuser de ne livrer dans Texaco qu'une "simple transcription des traditions orales populaires antillaises " ("Chamoiseau lui-même reconnaît qu'il est plus "marqueur de paroles" qu'écrivain ») et, pis, de les « intellectualiser » dans un roman « illisible » ${ }^{4} .$. De même, une partie de la critique a lu Chronique des sept misères comme un «roman ethnographique » : autant de lectures naïves amenant l'écrivain à expliciter son projet ${ }^{5}$.

11 L'un des aspects de ce projet, on l'a compris, est l'attention portée aux « Maîtres de la parole ", dont la disparition est le symbole d'« un monde finissant " face à des "mémoires indifférentes" (Chamoiseau, 1988a, 212). Avec le monde de la plantation disparaît «l'écosystème » du conteur, qui progressivement ne trouve plus de tribunes. Solibo « tenait à inscrire sa parole dans notre vie ordinaire, or cette vie n'en avait plus l'oreille, ni même de ces creux où s'éternise l'écho [...] son espace se réduisait. Des autorités de l'action culturelle avaient souvent sollicité sa participation à des spectacles de conteurs de scène, mais Solibo, craignant cette sorte de mise en conservation où l'on quittait la vie pour un cadre d'artifice, avait prétexté de mystérieuses obligations » (ibid., 208) ; " piège folklorique » estimait aussi Glissant, comme avant lui Aimé Césaire. Le créole "défaille", la parole est supplantée par l'écriture dans la logique de la francisation, écrit Chamoiseau dans "Que faire de la parole ?", sa contribution à Écrire la "parole de nuit»; "demeurent, pour l'écrivain, des lambeaux de mémoire orale, disséminés à travers le pays, des bouts de contes, des bribes de comptines, des éclats de titimes, des haillons de paroles diverses " (1994a, 155). Ina Césaire et Marie-Thérèse Lung-Fou, parmi d'autres, s'attachent à recueillir des contes, mais là n'est pas l'essentiel selon Chamoiseau. "Laisse la tradition, pitite », conseille Solibo, pour qui les contes ne sont que les feuilles en train de tomber, «laisse la tradition, et surveille la racine... » (1988a, 60). Le « don de parole » est un « don de souvenir» (ibid., 75), un don de mémoire.

Vous êtes fâché parce que vous voulez dire à Solibo Solibo baille-nous [donne-nous] parole des contes sur compère Tigre et sur compère Lapin sur Diable Ti-Jean et Nanie-Rosette mais Solibo ferme la bouche dessus et il dit Solibo qu'il n'est pas un bajoleur qu'il n'est pas là ce soir pour donner des leçons ou pour faire rire kia kia kia kia en faisant des tours et des détours flip-flap aléliron-viré blo par-devant frip ! glisse par-derrière tototo ? tototo ? qui est là ? blogodo c'est Solibo qui ne parle pas ce soir derrière une calebasse ou derrière un Tigre que pièce nègre n'a jamais vu [...] par-ici et ce que je vois c'est rien sinon des nègres z'habitants pas même malins comme compère Lapin [...] C'est Solibo qui hèle debout là dans le pays [...] et qui dit le paysage [...] qui dit hélez le paysage jusqu'à la soif d'un tafia et voir la vérité vue et commencer à sarcler la vérité vue si je dis Fonds-Massacre ? [...] personne ne sait qui a saigné là comme du sirop-là si c'est des nègres ou des crapauds.

(Chamoiseau, 1988a, 220-221).

12 Solibo ne donne pas des contes traditionnels, il veut redonner la mémoire à son auditoire, la mémoire de sa propre histoire. Chez Glissant (1981, 233), Fonds-Massacre 
est un lieu inconnu des Martiniquais, symbole de leur méconnaissance de l'histoire antillaise, qui empêche la population d'accéder à une véritable conscience collective. On reviendra sur l'idée de "dire le paysage" et de «fouiller le pays" (Chamoiseau, 1988a, 222).

13 Le Marqueur de paroles, en passeur de mémoire et en "prétendu ethnographe", recueille son matériau sur le terrain, auprès des djobeurs dans Chronique, auprès du conteur et de son public dans Solibo, auprès de Marie-Sophie Laborieux et d'autres habitants du quartier dans Texaco - texte dans lequel le Marqueur recueille aussi des matériaux écrits, puisqu'il reçoit les « notes de l'urbaniste » et surtout les « Cahiers » de sa principale « informatrice », qui elle aussi a tenté d'écrire la parole.

Cela me rapprocha d'elle qui, toute sa vie, avait poursuivi la parole de son père, et les mots rares de Papa Totone, et les bribes de nos histoires que le vent emportait comme ça, au fil des terres. C'est pourquoi elle me confia ses innombrables cahiers, couverts d'une écriture extraordinaire, fine, vivante de ses gestes, de ses rages, ses tremblades, ses taches, ses larmes, de toute une vie accorée en plein vol. Confondu d'avoir la charge de tels trésors, je les numérotai, cahier par cahier, page par page, je scotchai les déchirures, recousis les feuilles éparses, et couvris chaque exemplaires d'un plastique protecteur. Puis je les déposai à la Bibliothèque Schœlcher [...] je me sentais faible, indigne de tout cela, inapte à transmettre un autant de richesses.

(ibid., 1992, 494-495).

14 Dans Biblique des derniers gestes, roman publié dix ans après Texaco, Patrick Chamoiseau met à nouveau en scène un personnage d'écrivain, qui recueille son matériau lors d'une veillée mortuaire (ou plutôt ante-mortem) exceptionnelle par sa durée (trente-trois jours) et de nature fabuleuse. Il se sent investi d'une véritable mission, comme l'indique l'interprétation qu'il donne de son arrivée à la veillée :

[Le mourant] ne me connaissait pas mais il sembla me reconnaître. Il me fit un signe ambigu de la main. Cela semblait signifier qu'il m'attendait comme il avait attendu la plupart de ceux qui se trouvaient là. Je n'en tirai aucune fierté, juste l'écrasement d'une charge que je ne sus identifier.

(ibid., 2002, 52).

15 Cette mission consiste à faire en quelque sorte la "chronique d'une mort annoncée ", mort d'un vieux combattant (Balthazar Bodule-Jules), mort d'un monde, mort des espoirs soulevés en Martinique par les mouvements de décolonisation. La particularité dans ce roman est que le Marqueur de paroles se trouve confronté cette fois à ce qu'on pourrait appeler un conteur muet, un langage silencieux. Au tout début du roman figure l'encouragement formulé par Isomène Calypso, "conteur", à celui qui est à nouveau identifié à l'auteur :

Vois la voie, mon Ti-Cham: il existe une parole qui est dite mais que personne n'entend; elle monte de nos abîmes pour dire ce que nous ne disons pas ou ne savons pas dire. Balthaz Bodule-Jules entendait cette voix dans les blesses de son âme, c'est pourquoi on pouvait le crier : l'Entendant justement.

(ibid., 2002, 15).

16 Au narrateur-écrivain-Marqueur de paroles de saisir cette parole qu'on n'entend pas, de recevoir le don silencieux d'un savoir à transmettre. 


\section{En deçà de la parole : mémoire du corps, mémoire des pierres, savoir enfoui}

17 Chez le mourant, pour paraphraser Benjamin, la vie vécue prend une forme dont l'écrivain peut s'emparer, l'inoubliable se révélant dans sa mimique et ses regards (1991). C'est ce qui se passe dans ce roman : l'assemblée réunie autour de Balthazar Bodule-Jules, et le narrateur-écrivain au premier plan, guettent les gestes, attitudes et émotions du vieillard dont ils cherchent à reconstituer la vie, vie légendaire pour autant qu'il a "rêvée autant qu'il l'a vécue » (Chamoiseau, 2002, 50). « Son corps et ses silences parlaient » commente le narrateur face au mourant, «comme s'il y avait eu là une mémoire particulière, une sapience " (ibid., 57-58). Cette fois, il s'agit donc de gestes et non plus de paroles, car l'auteur veut transmettre la parole perdue, l'inaudible, "investir ces zones impénétrables du silence où le cri s'est dilué » (Chamoiseau, Bernabé et Confiant, 1989, 38). Le narrateur dit se trouver au cœur d'une " chorale silencieuse ", d'un " assourdissant ouélélé de silence », obligé de grapiller des «miettes de vocables ", des «bribes de murmures» (Chamoiseau, 2002, 140, 111, 75)... L'« étonnante assemblée " qu'il découvre réunie autour du vieil homme n'est pas ordinaire - «trâlée de figures échappées aux oubliettes du temps, silencieuses et discrètes » (ibid., 284) :

Je croyais côtoyer des spectres des temps anciens, fantômes d'époques invalidées, détenteurs des sapiences désapprises depuis déjà longtemps. Ces hommes, ces femmes, ces odeurs, ces manières, cette posture des corps, les sonorités du bavardage créole, ces discours de silences, ces murmures solitaires, me plongeaient dans un bain d'étrangeté radicale et de très vieille proximité. Ces étrangers relevaient du réel oublié, celui que $\mathrm{M}$. Balthazar Bodule-Jules nommait sans rire depuis déjà longtemps : le pays enterré.

(ibid., 51).

Cette formule, par ailleurs immédiatement compréhensible (il s'agit du pays d'antan, de personnages du passé), renvoie directement à la thématique de l'enfoui, de l'«endessous ». L'Éloge de la créolité préconise la "minutieuse exploration» de "ce nousmêmes enfoui sous la francisation", démarche assimilée aux fouilles archéologiques (Chamoiseau, Bernabé et Confiant, 1989,22). Glissant invitait à une démarche comparable en désignant " la mémoire historique » comme "trop souvent raturée »: "l'écrivain antillais doit "fouiller" cette mémoire, à partir de traces parfois latentes qu'il a repérées dans le réel » (Glissant, 1981, 227-228). Pour Glissant, la terre garde témoignage du passé. "Notre paysage est son propre monument: la trace qu'il signifie est repérable par-dessous. C'est tout histoire » écrit-il dans le Discours antillais (ibid., 32), d'où une dénonciation des transformations que subit le pays : bétonisation, pollution... Sous couvert de progrès, le paysage est transformé et sa « lecture », son interprétation, sont rendues impossibles, de même que l'imaginaire martiniquais est recouvert par le rêve d'une modernité française, modernité "anesthésiante " (Chamoiseau, 1997a, 75-76) qui renforce la rupture avec le passé antillais. Le début de Biblique des derniers gestes est la description de cette modernité qui caractérise le «pays officiel ", et d'une population égarée dans le spectacle d'une réalité appauvrie, "passée à côté de son cri " disait Aimé Césaire dans le Cahier d'un retour au pays natal, " étrangement bavarde et muette» $(1939,9)$. En contraste avec elle, Chamoiseau décrit une communauté mythique dotée de la mémoire collective que ne possèdent pas ceux du «pays réel », mémoire perdue ou « désapprise ». Le citoyen du "pays réel » est 
assourdi par des bruits parasites (dont sont en grande partie responsables les médias); celui qui cherche à entendre la voix du "pays d'avant» ou "pays enterré » est confronté au silence ou à des murmures, des bribes qu'il ne peut que « réinventer ».

Faisant le constat de la défaillance de la "mémoire du souvenir », Chamoiseau explore dans ce roman une mémoire "de bas niveau», «mémoire de l'empreinte» (Bonniol, 2007). Son personnage d'écrivain désenfouit la «mémoire vraie» de son peuple en déchiffrant non pas le paysage mais des gestes et des stigmates, en décryptant ce qu'on pourrait appeler un paysage corporel, d'ailleurs en correspondance avec le paysage physique, l'un et l'autre marqués par les tracesblessures encore non refermées du passé : «lèpre » aux poignets et chevilles frappant des descendants d'esclaves martyrisés, «tête-fendue» d'avoir à contenir une "mémoire impossible à loger» (Chamoiseau, 2002, 486) (concrétisation de «l'hypermnésie » derridienne ?), «blesse » provoquée par une chute dans un lieu où l'on pratiquait le «supplice du tonneau »... La «blesse» est un "mal mystérieux, quasiment invisible, qui pouvait prendre tout le monde » au hasard d'une promenade dans l'un de ces lieux « de mémoire effroyable comme il y en avait un peu partout dans le pays » (ibid., 476-477) ; ces lieux maudits provoquent diverses calamités chez ceux qui les fréquentent, tel ce domaine d'où un cachot oublié diffuse une puissance mortifère ; ils doivent être apaisés par "un geste de mémoire " (ibid., 487), de recueillement, «l'inclination d'une conscience au-dessus du crime abandonné » (ibid., 523). Le paysage et les traces du passé qu'il contient apparaissent cependant comme illisibles au noninitié ; la parole, quand elle est signifiante, comme inaudible; ils sont accessibles à Balthazar Bodule-Jules grâce au savoir transmis par "Man l'Oubliée ", une "femme de force ", une Mentô, qui lui apprend à lire le paysage et à soigner les corps (et les âmes), et accessibles au narrateur par l'initiation que représentent son "geste de mémoire ", sa propre pratique de recueil/« recueillement».

On retrouve le personnage d'initiatrice qu'est «Man l'Oubliée » dans le roman suivant, Un dimanche au cachot, dans lequel le narrateur imagine qu'elle a été enfermée par un colon dans un trou creusé sous une butte. Le passé suinte de la roche et c'est en se laissant imprégner par l'atmosphère du lieu qu'il pense parvenir à accéder à l'expérience de cette femme, à son "intériorité ». Le narrateur quant à lui appartient au monde (fictif) contemporain (il est travailleur social, un double là encore du romancier dont c'est l'autre métier) et c'est pour venir en aide à une orpheline qui s'y est réfugiée, hébétée de malheur et d'ignorance de son passé, qu'il s'enferme une journée dans ce cachot - double d'un cachot réel devant lequel les journaux locaux ont photographié l'auteur à la sortie du roman : comme toujours, Chamoiseau promène son lecteur du réel à l'imaginaire, de la métaphore à la fiction... Dans ce roman publié en 2007, il reprend la structure en deux temps de Biblique des derniers gestes, un prologue qui désigne au lecteur le pays réel et dont l'écriture frappe par sa trivialité, et le corps du récit, exploration du passé esclavagiste par un narrateur doué de la puissance " prophétique » de son imagination, en présence de l'une de ces figures de la prescience malheureuse du passé, fillette dans Un dimanche au cachot, "vieux corps » dans Biblique des derniers gestes, femme tombée folle chez Glissant (Marie Celat) ou chez Schwarz-Bart - femmes allongées sur le sol, au niveau des herbes et des fourmis. Ces personnages sont en relation avec le " pays enterré » et une correspondance évidente apparaît entre le cachot et les "caches" des romans de Glissant. La terre recèle une mémoire souterraine que l'écrivain antillais doit fouiller - ce à quoi l'invite le Discours antillais. 
C'est en fouillant que Mathieu Béluse, dans Mahagony (roman de Glissant publié en 1987), retrouve l'écriture clandestine de l'esclave Hégésippe, un de ces «marrons du syllabaire » décrits par Jean Fouchard. Hégésippe est un « vieux houeur » qui a fouillé la terre pour enfouir son témoignage (inscrit sur une écorce), dans un pays où les esclaves n'ont pas laissé d'écrits ${ }^{6}$. Lydie Moudileno a souligné l'importance dans Mahagony de la révélation d'un système de caches qui fonctionnent comme "archive" au sens foucaldien, liée à la démarche "archéologique» qui caractérise l'un des modes d'approche de la culture antillaise par Glissant et par les écrivains de la Créolité (Moudileno, 1997, 113-140). Le paysage en témoin muet capable de suggérer, la terre parce qu'elle recèle des caches et des traces, sont une archive du passé.

21 Le trope de la cache apparait dans plusieurs textes de Chamoiseau et dans la Jarre d'or de Confiant. Le trésor enfoui est un motif récurrent dans la tradition orale antillaise, ces jarres enterrées par les riches planteurs avec leurs biens les plus précieux au moment de révoltes d'esclaves; dans ces caches auraient parfois été précipités, dit la légende, les esclaves chargés de les creuser, enterrés avec leur secret et transformés en gardiens éternels du trésor. Cette légende apparaît chez Confiant mais aussi chez Chamoiseau dans Chronique des sept misères : elle hante l'un des personnages principaux, Pierre-Philomène Soleil, qui s'allonge à plat ventre dans l'herbe pour se soûler « de l'étrange musique des remontées de la jarre " (Chamoiseau, 1986,132). Afoukal, le zombi qu'il entend dans ses rêves, ne lui apprend pas le secret de l'emplacement de la jarre, mais lui laisse un témoignage du temps de l'esclavage, paroles qui lui permettent de remonter «sa propre mémoire fendue d'oubli comme une calebasse et enterrée au plus loin de lui-même» (ibid., 133). De même dans le roman de Confiant. Signalons toutefois que, comme l'écorce trouvée par le personnage de Glissant (et son double romanesque), le trésor de la Jarre d'or n'est ni d'or ni de paroles mais d'écritures : c'est "la jarre de livres bannis", contenant livres ésotériques et livres d'histoire de l'esclavage, mis sous scellés pour ne pas menacer la paix sociale. Mais «l'archive » est surtout contenue chez Confiant dans la langue créole $(1982,35)$ : là se trouve aussi un trésor enfoui'. Même si ces amoureux de l'écrit que sont Confiant, Chamoiseau et Glissant, réintroduisent dans ces "terres d'oralité " des archives écrites introuvables (témoignages d'esclaves), confisquées (dans des jarres enfouies ou dans des bibliothèques fermées à clé) ou improbables (les «Cahiers" de «l'informatrice » dans Texaco), la tradition orale constitue, dans la démarche « d'exploration minutieuse de ce nous-mêmes enfoui sous la francisation ", un véritable trésor, parce que porteuse du créole d'antan et de l'imaginaire qui lui était lié.

Terminons ce cheminement par l'incipit et l'excipit de l'Esclave vieil homme et le molosse, conte poétique de Chamoiseau dans lequel le narrateur fait entendre sa voix dès les premières lignes :

Ainsi, m'est parvenue l'histoire de cet esclave vieil homme. Une histoire à grands sillons d'histoires variantes [...] Ici, soucieux de ma parole, je ne saurais aller qu'en un rythme léger flottant sur leurs musiques.

(Chamoiseau, 1997b, 17).

Pourtant le texte se termine, comme Texaco («Résurrection »), par l'explication par le Marqueur de paroles de la genèse de son récit: "Très souvent de vieux-nègres viennent m'exhiber l'antan [...] Il[s] m'offr[e]nt d'antiques objets ; me montr[e]nt des choses d'âge ; me propos[e]nt leur vie à rédiger et leurs exploits à raconter " (1997b, 141). L'un d'eux a fouillé les bois et trouvé une pierre couverte de pétroglyphes amérindiens (cette autre voix perdue à retrouver dans une littérature créole); en 
fouillant encore, "sans doute à la recherche de ces trésors qui nous tracassent les rêves » (ibid., 143), le vieil homme a trouvé des os, qu'il a apportés au Marqueur, des os " chargés » d'« un cri muet sans sortie » (ibid., 144); dès lors celui-ci y a pensé jusqu'à l'obsession, jusqu'à écrire cette histoire, celle de la fuite d'un nègre marron. "J'essaierai de modeler mon vieux-bougre dans un langage de conte et de souffle de course. Un langage qui dirait sa parole en le signalant muet » (ibid., 145). L'histoire donnée pour une variante de conte est bel et bien l'œuvre d'un écrivain, qui s'est d'abord donné à voir comme narrateur pour mieux s'effacer comme auteur, avant de se présenter comme le récipiendaire d'une parole inaudible, aussi bien que d'un don qu'il ne peut ni refuser ni conserver pour son seul profit.

\section{Conclusion}

24 À une époque où le devoir de mémoire était sur toutes les lèvres et où la quête de légitimité et d'« authenticité » en littérature passait par une filiation réinventée avec le conteur, les romanciers antillais ont été nombreux à se présenter ou à présenter un double romanesque, dans une mise en évidence de l'acte de narrer, comme un conteur, un passeur, un marqueur, répondant à un don de paroles - lesquelles avaient d'ailleurs moins à voir avec la tradition orale qu'avec la mémoire historique. Pourtant l'affirmation s'est souvent trouvée niée dans le même temps, avec le constat d'une rupture dans la transmission, d'une mémoire perdue, qui a amené Glissant à élaborer l'idée forte de "vision prophétique du passé », reprise par Chamoiseau, qui valorise de façon croissante les intuitions, l'imaginaire de l'écrivain - à qui il reste finalement à "tout imaginer »- et la valeur heuristique de la littérature ${ }^{8}$. Dans Biblique des derniers gestes, la figure du Marqueur de paroles s'efface d'ailleurs devant celle du " guerrier de l'imaginaire ». Mais la scénographie complexe que l'auteur met en œuvre révèle toujours sa volonté de faire comme si la communication avec les anciens n'avait pas été interrompue. Don de cahiers manuscrits, de messages sur des écorces enfouies dans la terre, de signes gravés dans la pierre, d'os, de grains d'or, de livres, don de mots, d'expressions corporelles, de gestes, de silences "chargés »: dans bien des textes de Glissant, Confiant, Chamoiseau, un don - don de mémoire - est à l'origine du récit. Le don en effet s'accompagne d'une charge et l'écrivain s'en acquitte par son récit, qui est un don analogue fait au lecteur. Les trois auteurs sont très attentifs à la réception de leurs textes par les lecteurs antillais; le discours de Chamoiseau sur ce point a évolué d'une vision du romancier dans le cercle, au plus près de ses lecteurs/auditeurs - une vision qui l'a conduit, comme Confiant et la plupart de leurs pairs au cours des vingt dernières années, à s'investir dans un " parler avec " et dans un " lire ensemble " ${ }^{9}$ - à une perspective plus élitiste ou plus mystique, plus proche de celle de Césaire ou de Glissant, selon laquelle le discours littéraire frappe les imaginations, "irradie ", incantatoire et opaque - mais en accord, toujours, avec la parole du conteur créole. 


\section{BIBLIOGRAPHIE}

BENJAMIN, Walter, 1991, Le Narrateur, Écrits français, Paris, Gallimard, coll. « Bibliothèque des

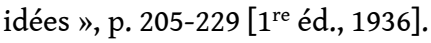

BERNABÉ, Jean, 1997, De l'oralité à la littérature antillaise : figures de l'Un et de l'Autre, in F. Tétu de Labsade, (éd.), Littérature et dialogue interculturel : culture française d'Amérique, Sainte-Foy, Presses de l'université Laval, p. 49-68.

BONNIOL, Jean-Luc, 2007, Les usages publics de la mémoire de l'esclavage colonial, Matériaux pour l'histoire de notre temps, $\mathrm{n}^{\circ}$ 85, p. 14-21.

CÉSAIRE, Aimé, 1939, Cahier d'un retour au pays natal, Paris, Présence africaine.

CÉSAIRE, Ina, 1976, Contes de mort et de vie aux Antilles, Paris, Nubia.

CÉSAIRE, Ina, 1989, Contes de nuits et de jours aux Antilles, Paris, Éditions Caribéennes.

CHAMOISEAU, Patrick, 1982, Manman Dlo contre la fée Carabosse, Paris, Éditions Caribéennes.

CHAMoISEAU, Patrick, 1986, Chronique des sept misères, Paris, Gallimard.

CHAMOISEAU, Patrick, 1988a, Solibo Magnifique, Paris, Gallimard.

CHAMOISEAU, Patrick, 1988b, Au temps de l'antan : contes du pays Martinique, Paris, Hatier.

CHAMOISEAU, Patrick, BERNABÉ, Jean, CONFIANT, Raphaël, 1989, Éloge de la créolité, Paris, Gallimard.

CHAMOISEAU, Patrick, CONFIANT, Raphaël, 1991, Lettres créoles. Tracées antillaises et continentales de la littérature : Haïti, Guadeloupe, Martinique, Guyane : 1635-1975, Paris, Gallimard, coll. « Folio ».

CHAMOISEAU, Patrick, 1992, Texaco, Paris, Gallimard, coll. « Folio ».

CHAMOISEAU, Patrick, 1994a, Que faire de la parole ? Dans la tracée mystérieuse de l'oral à l'écrit, in R. Ludwig (éd.), Écrire la " parole de nuit ». La nouvelle littérature antillaise, Paris, Gallimard, coll. « Folio », p. 151-158.

CHAMOISEAU, Patrick, 1994b, 2002, Le dernier coup de dent d'un voleur de banane, in R. Ludwig (éd.), Écrire la " parole de nuit ». La nouvelle littérature antillaise, Paris, Gallimard, coll. « Folio », p. 29-38.

CHAMOISEAU, Patrick, 1994c, Chemin d'école, Paris, Gallimard.

CHAMOISEAU, Patrick, 1997a, Écrire en pays dominé, Paris, Gallimard, coll. « Folio ».

CHAMOISEAU, Patrick, 1997b, L'Esclave vieil homme et le molosse, Paris, Gallimard, coll. « Folio ».

CHAMOISEAU, Patrick, 2002, Biblique des derniers gestes, Paris, Gallimard, coll. « Folio ».

CHAMOISEAU, Patrick, 2007, Un dimanche au cachot, Paris, Gallimard.

CHAMOISEAU, Patrick, 2011, Le papillon et la lumière (conte philosophique), Paris, Philippe Rey. CHANCÉ, Dominique, 2000, L'auteur en souffrance. Essai sur la position et la représentation de l'auteur dans le roman antillais contemporain, 1981-1992, Paris, PUF.

CONFIANT, Raphaël, 1982, Charte culturelle créole (avec le GEREC), Schœlcher, Éditions du Centre universitaire Antilles-Guyane.

CONFIANT, Raphaël, 1988, Le Nègre et l'Amiral, Paris, Grasset. 
CONFIANT, Raphaël, 1994a, Questions pratiques d'écriture créole, in R. Ludwig (éd.), Écrire la « parole de nuit ». La nouvelle littérature antillaise, Paris, Gallimard, coll. « Folio », p. 171-180.

CONFIANT, Raphaël, 1994b, Fils du diable en personne, in R. Ludwig (éd.), Écrire la « parole de nuit ». La nouvelle littérature antillaise, Paris, Gallimard, coll. « Folio », p. 39-51.

CONFIANT, Raphaël, 1995a, Les Maîtres de la parole créole, Paris, Gallimard. [Présentation et transcription par Raphaël Confiant, photographies de David Demoison, textes recueillis par Marcel Lebielle].

CONFIANT, Raphaël, 1995b, Contes créoles des Amériques, Paris, Stock.

CONFIANT, Raphaël, 2010, La Jarre d'or, Paris, Mercure de France.

FOUCHARD, Jean, 1988, Les Marrons du syllabaire, Port-au-Prince, Éd. Henri Deschamps.

GATES, Henry Louis Jr., 1988, The Signifying Monkey: A Theory of African-American Literary Criticism, New York, Oxford University Press.

GLISSANT, Édouard, 1981, Le Discours antillais, Paris, Gallimard.

GLISSANT, Édouard, 1987, Mahagony, Paris, Seuil.

GLISSANT, Édouard, 1988, Un marqueur de paroles [préface à la réédition de Chronique des sept misères], Paris, Gallimard, p. 3-6.

LESNE, Anna, 2013, S'écrire aux Antilles, écrire aux Antilles - Écrivains et anthropologues en dialogue, L'Homme, n 207-208, « Un miracle créole? », Paris, Éd de l'EHESS.

LUNG-FOU, Marie-Thérèse, 1979, Contes créoles, 3 vol., Fort-de-France, Désormeaux.

MOUDILENo, Lydie, 1997, L'écrivain antillais au miroir de sa littérature, Paris, Karthala.

PÉPIN, Ernest, 1992, L'Homme-au-bâton, Paris, Gallimard.

PINEAU, Gisèle, 1993, La Grande drive des esprits, Paris, Le Serpent à Plumes.

SCHWARZ-BART, Simone, 1979, Ti-Jean L'Horizon, Paris, Seuil.

\section{NOTES}

1. L'Homme-au-bâton est une variation sur une légende urbaine partie d'un fait divers, les crimes sexuels d'un assassin rapidement assimilé dans la rumeur au « dorlis ", sorte d'incube antillais. 2. Voir CONFIANT (1995b) et CHAMOISEAU (1988b) (transcription de contes) ; CONFIANT (1995a) (ouvrage sur les conteurs) ; CONFIANT (1994b) ; CHAMOISEAU (1994b et 2011) [écriture de contes]. 3. Pour une définition de l'oraliture, voir BERNABÉ $(1997,51-56)$. Pour BERNABÉ, linguiste et coauteur de l'Éloge de la créolité avec CHAMOISEAU et CONFIANT, « la notion de littérature orale est une pure contradiction ", il reprend donc le terme d'oraliture inventé selon lui « par les ethnologues africanistes dans les années 1960 » (il ne fait pas référence à Zumthor). Il l'utilise pour désigner les productions orales des genres "structurés selon une textualité mémorielle ", "genres mnémoniques tels que le mythe, le conte, l'épopée, le proverbe, le dicton, [etc.]»; mais le terme d'oraliture sert aussi selon lui à désigner dans le contexte antillais la "parole de nuit ", parole communautaire des esclaves sur la plantation. Oralité et oraliture se distinguent essentiellement par leur lien à la mémoire à court terme pour la première et à la mémoire à long terme pour la seconde ; l'oraliture, comme l'écriture, est un "conservatoire de la parole », mais fonctionne selon un " circuit bouche-oreille in praesentia et en temps réel» et est l'expression du collectif, 
tandis que l'écriture (circuit œil-main) est « le lieu par excellence d'individuation de la parole conservée ».

4. «Aigreurs d'estomac », critique parue dans France-Antilles le 12/12/1992.

5. Sur l'identification de l'auteur avec un ethnographe, voir LESNE (2013).

6. Il faut noter en effet que tandis qu'aux États-Unis le genre des slave narratives s'est largement développé au XIX siècle au point, selon Henri Louis GATES (1988), de fonder l'écriture « africaineaméricaine ", ce n'est que par la traduction que les francophones ont accès aux témoignages d'esclaves.

7. Dans le Nègre et l'Amiral, confiAnT écrit: «[Amédée Mauville, le Mulâtre aspirant écrivain] cherchait ce mot (ou cette parcelle de phrase) comme d'autres une jarre d'or enterrée du temps de l'esclavage " $(1988,50)$; en lui redonnant accès au créole, à un créole plein et vivant des classes populaires, la négresse Philomène provoque chez lui le déclic de l'écriture, qui coïncide avec un geste, le don d'un grain d'or de son collier ; le thème de la Jarre d'or s'ébauche déjà.

8. Un vaste métatexte est développé dans le roman sous forme de commentaires dispersés, les «notes d'atelier et autres affres » : "Tout est faux et incertain quand on s'alimente aux paroles sillonnantes et aux mémoires des gens. Mes notes et mes brouillons relus maintenant n'ont plus grand sens et ne m'offrent nulle vérité. Ils n'attestent que de mon intention de recueillir des fluidités de perception. Seul l'effet à trouver peut me rapprocher de la réalité. Seul l'effet doit compter et, pour une fois, je m'y résigne d'une manière consciente. L'Écrire n'a rien à voir avec la vérité, ni avec le réel : l'Écrire n'est qu'une quête de la vie, la plus libre et la plus folle des quêtes, donc la plus tressaillante de cette vie même qu'elle cherche... » (Chamoiseau, 2002, 138).

9. Le rapport dynamique établi entre le conteur et son public, entre les romanciers antillais et leurs lecteurs, est l'autre versant de la question traitée ici ; un article sur ce thème est paru en 2014: A. LESNE, 2014, La construction d'une scène littéraire antillaise. Médiations et réappropriations, Ethnologie française, $\mathrm{n}^{\circ} 4$, «Ethnologie[s] du littéraire ».

\section{RÉSUMÉS}

Nombre de romanciers antillais cherchent à prendre le relais des conteurs, se mettant en scène comme mandatés ou attachés à recueillir et transmettre des histoires. En quête de légitimité, et tout en valorisant par ailleurs la fiction et le romancier, ils se font passeurs de mémoire, relais d'autres voix ou de la parole collective; et quand les paroles sont inaudibles, quand la mémoire est perdue et que l'écrivain doit finalement «tout imaginer », il est encore présenté, chez Patrick Chamoiseau surtout, comme recevant un don - manuscrits et objets chargés d'histoire, mémoire des corps et mémoire des pierres - auquel il répond par son récit.

Numerous French Caribbean authors aim to continue the storyteller tradition. They stage themselves as if they were mandated to, or in charge of, gathering and transmitting stories. In search of legitimacy, and while otherwise praising fiction and the novelist, they pose as the passers on of memory, the relays of other voices and of the collective narrative. And when the words become inaudible, when memory is lost and the author finally must "make up everything," he is still presented, in particular in the works of Patrick Chamoiseau, as if he had received a gift - manuscripts or objects loaded with history, the memory of stones and bodies - that he responds to in his tale. 
INDEX

nomsmotscles Antillais

Thèmes : anthropologie (Amérique), littérature orale

Keywords : Narrator, Authoriality, Legitimacy, Collective Voice, Memory, Slavery

Mots-clés : narration, auctorialité, voix collective, mémoire, esclavage

Index géographique : Antilles

\section{AUTEUR}

ANNA LESNE

Institut d'ethnologie méditerranéenne, européenne et comparative, New York University à Paris 